\title{
Study on the Method for Comprehensive Evaluation of Complex System Maintainability
}

\author{
YANG Ming-hua1, WANG Guo-gang1, HAN Xiao-ping1, LIANG Tao2
}

Department of Arms Engineering, Academy of Armored Force Engineering, Beijing 100072, China; Troop No. 63880 of PLA, Chenzhou 423026, China

Keywords: Maintainability; Influence Degree; Correlation Matrix; Comprehensive Evaluation

\begin{abstract}
In order to assess the maintainability of weapon system on the system-level more scientifically and accurate, the concept of "Influence degree" is introduced into this study under the consideration of the relational degree between the influence of components on system and assessment indicators. By combining the weight of each component, we get the integrated system maintainability index. Then the comprehensive assessment of the maintainability of weapon system is made by computing the influence degree of each component's assessment indicators on system maintainability. The assessment results show that this method is more credible and the information obtained through the maintainability assessment process has great significance for system design and maintainability improvement.
\end{abstract}

\section{Introduction}

The degree of complexity of modern weapons and equipment continues to improve, the huge system, versatile and expensive, maintenance of quality as one of its important characteristics, require scientific and accurate evaluation. Comprehensive evaluations of weapon system maintenance repairs to understand its true level, to improve the system design are of great significance. At present, domestic and international aspects of the comprehensive evaluation of the maintenance carried out a series of exploration and research. Such as Cai Jing and Zuo Hongfu Nanjing University of Aeronautics and Astronautics presented computing expert assessment in determining maintenance products on the basis of the evaluation factors to achieve their weight maintainability comprehensive evaluation method [1], the entire evaluation process clear, however, there are still difficult to determine exactly how the experts on weight; National Defense Science and Sun Quan Yan Pengfei credibility weighted fusion model based on the calculation results of the evaluation of the maintainability index, based on a small sample test [2], the method does not consider the relationship between indicators and only applies to the evaluation of the maintenance time and other quantitative indicators, has certain limitations; Professor MF Wani evaluation noted among the earliest American maintainability evaluation of the relationship between the existence of mutual influence, Shanghai Jiaotong University's Chen Lu et maintainability Relational used during the evaluation method [3], taking into account the relationship between the evaluation index, but only for simple component maintenance was evaluated, there is no comprehensive study of complex evaluation system maintainability. To solve the above problem, in order to obtain a more scientific and accurate evaluation results, based on the above studies, this paper introduces "degree of influence" used to evaluate a new type of weapon systems and equipment serviceability.

\section{2. evaluation of ideas}

Refers to the degree of influence on the evaluation of the impact evaluation of object maintenance level in that ideal state indicators (evaluation score of 10), the obtained composite index maintenance evaluation system, with respect to the maintenance of the growth rate of the real system evaluation Composite Index. In this paper, based on the degree of influence the degree of influence $\Delta$ is defined formula:

$$
\Delta=\frac{\operatorname{per}^{\prime}(W Q)-\operatorname{per}(W Q)}{\operatorname{per}(W Q)}
$$


Where: $\operatorname{per}^{\prime}(W Q)$ is Maintainability Index System Index under ideal conditions; $\operatorname{per}(W Q)$ is the actual system maintainability index. $\Delta$ is a dimensionless quantity, the $\Delta$ greater the level of maintenance of the system shows that the larger the lower the evaluation of the object can be room for improvement.

In order to obtain a comprehensive evaluation index system maintenance, system-level first-class top-down decomposition of the components, the various components of the system is obtained based on the existing right of empirical data in the system engineering evaluation of heavy maintenance, through indicators related degree and have to Figure build weapons systems evaluation model consisting of components, using correlation matrix product and the formula [4] the method is based on the maintenance of the various components were evaluated. The formula for the volume and type of matrix $A=\left(a_{i j}\right)_{n \times n}[3]$

$$
\operatorname{per} A=\sum_{j_{1} j_{2} \cdots j_{n}} \prod_{i=1}^{n} a_{i j_{(i)}}
$$

Relevance of the system for maintenance model for evaluation of matrix product and style, each element can be considered the relationship between them and the associated matrix, and as a comprehensive evaluation of maintainability index. By seeking correlation matrix product and the type of complex systems maintenance assessment is a simple evaluation method, not only consider the impact of index itself, but also consider the impact of the relationship between the indexes, can fully evaluate the various components maintainability. Under normal circumstances the system's maintainability index values also influenced by the characteristics of the system architecture. Since the weapon system repair process is almost no mutual influence between the various subsystems can be approximated as independent, so the maintenance of the structural features of the Index of this article will not be considered.

On the basis of evaluation for repair composite index were calculated on the degree of influence of each of the components of each index, by analyzing the impact of the complex system used to evaluate the level of maintenance.

\section{3. evaluation process}

\subsection{Maintenance Evaluation Index Selected}

As an important part of weapons, weapons systems and equipment is one of the largest and most complex subsystems, and its good level of service is an important guarantee for the entire equipment to meet the reliability requirements [5]. Weapons systems including: fire section $(\mathrm{H})$, the fire control system $(\mathrm{K})$ and integrated information systems $(\mathrm{Z}) 3$ parts.

In weapon system design criteria and requirements as the basis, select accessibility, assembly and disassembly, standardization and interchangeable degree detection and diagnosis of performance and human factors engineering five indicators as weapon systems and their components maintenance evaluation index composed of [6], and were replaced with A, B, C, D, E.

\section{2 parts maintainability index calculation}

Because of the relationship between each index are not independent of each other, with varying degrees of mutual influence, referred to as "associate degree" [7]. Maintainability evaluation in reference to the provisions of the US standard score [8], the use of 0,2,4 score standard to represent association between the degree of each index. 4 shows a strong correlation between indicators; 2 indicates a weak correlation between indicators; 0 indicates no correlation between the index, i.e., there is no influence. Table 1 shows the degree of association selected Maintainability Index. 
Table 1 maintainability evaluation related degree

\begin{tabular}{cccc}
\hline evaluation & \multicolumn{3}{c}{ associate degree } \\
\cline { 2 - 4 } index & 4 & 2 & 0 \\
\hline A & B & D,E & C \\
B & A & E & C,D \\
C & B & A,E & D \\
D & E & -- & A,B,C \\
E & -- & A,D & B,C
\end{tabular}

As can be seen from Table 1, not affect the relationship between the symmetrical indexes is directional. For example: reach ability detection diagnostic performance related degree is 4 , but the detection and diagnosis of performance associated with the degree of accessibility is 0 , meaning that there is no impact on the performance of detection and diagnosis of accessibility.

There are plans to use the network represents a service evaluation model part [9], shown in Figure 1

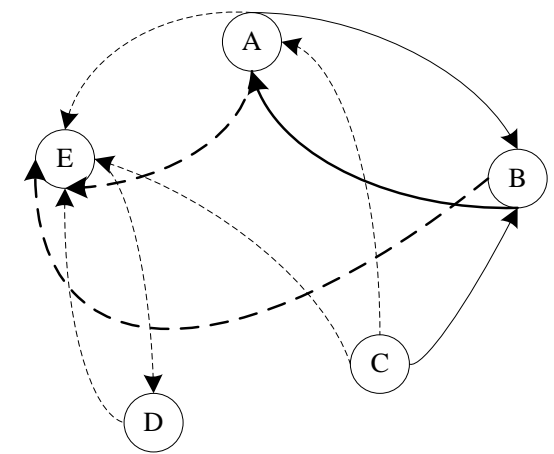

Figure 1 Composition Assessment between component repair a directed graph model

Each node represents the maintenance of the index evaluation. The solid line indicates a strong correlation between the indexes, such as the line segment A to B, point A represents the relationship $\mathrm{B}$, i.e. a degree of relevance of the $\mathrm{B}$ size. Dashed line indicates a weak correlation between the indexes, there is no connecting lines indicates no correlation between the two indicators.

In the evaluation process of maintenance, the application of the plot and style can be better relations between the various indicators characterize it, thus solves the problem of mutual influence between indicators [9]. Table 2 is a weapon system components integrated weight matrix $v_{1} \sim v_{5}$ for maintainability index score value, the value of non-diagonal correlation between the degrees of maintainability index.

Table 2 weapon system components of a comprehensive weight matrix

\begin{tabular}{cccccc}
\hline $\begin{array}{c}\text { Indicators } \\
\text { / Score }\end{array}$ & $\mathrm{A}$ & $\mathrm{B}$ & $\mathrm{C}$ & $\mathrm{D}$ & $\mathrm{E}$ \\
\hline $\mathrm{A}$ & $v_{1}$ & 4 & 0 & 2 & 2 \\
$\mathrm{~B}$ & 4 & $v_{2}$ & 0 & 0 & 2 \\
$\mathrm{C}$ & 2 & 4 & $v_{3}$ & 0 & 2 \\
$\mathrm{D}$ & 0 & 0 & 0 & $v_{4}$ & 2 \\
$\mathrm{E}$ & 2 & 0 & 0 & 2 & $v_{5}$ \\
\hline
\end{tabular}

Maintainability index score $v_{1} \sim v_{5}$ are based on the post-Jack system for maintenance of weapon system simulation test, and then determined based on the analysis of engineering experience. After evaluation of the accessibility to (A) the scoring process as an example, the establishment of "0" to "4" 5 rating scale, the use of Jack system reachability analysis tools to establish physical contact range of packages area network, the regional analysis of the specific reachability scoring criteria shown in Table 3 
Table 3 reachability scoring criteria

\begin{tabular}{ll}
\hline \multicolumn{1}{c}{ Scoring criteria } & scores \\
\hline Visual reachable, accessible and adequate working & 4 \\
space & \\
Satisfying the above condition of at least one of & 2 \\
Not satisfy & 0 \\
\hline
\end{tabular}

Similarly you can get another evaluation of the evaluation score. According to equation (2) to calculate the maintainability index, let $\mathrm{H}, \mathrm{K}$, maintainability index $\mathrm{Z}$ are three components $\operatorname{per}(H), \operatorname{per}(K), \operatorname{per}(Z)$. That maintainability index components are as follows:

$$
\operatorname{per}(i)=v_{1}\left\{\begin{array}{llll}
v_{2} & 0 & 0 & 2 \\
4 & v_{3} & 0 & 2 \\
0 & 0 & v_{4} & 2 \\
0 & 0 & 2 & v_{5}
\end{array}\right\}=v_{1} v_{3}\left\{\begin{array}{ccc}
v_{2} & 0 & 2 \\
0 & v_{4} & 2 \\
0 & 2 & v_{5}
\end{array}\right\}=v_{1} v_{3} v_{2}\left(v_{4} v_{5}+4\right)
$$

Through the maintainability index of each of the components simulation test analysis, combined with practical experience in the maintenance of the components identified evaluation score, as shown in Table 4

Table 4 weapon system maintainability index of each component evaluation data table

\begin{tabular}{llllll} 
& A & B & C & D & E \\
\hline$H$ & 2 & 4 & 2 & 4 & 2 \\
$Z$ & 2 & 2 & 2 & 2 & 2 \\
$\mathrm{~K}$ & 2 & 4 & 2 & 0 & 4 \\
\hline
\end{tabular}

Finally determined based on the data in Table $4 \mathrm{H}, \mathrm{K}, \mathrm{Z}$ of the maintenance of the weight matrix are integrated as shown in Table 5, Table 6, Table 7

Table $5 \mathrm{H}$ maintainability comprehensive weight matrix

\begin{tabular}{cccccc}
\hline $\begin{array}{c}\text { Indicators / } \\
\text { Score }\end{array}$ & A & B & C & D & E \\
\hline A & 2 & 4 & 2 & 4 & 2 \\
B & 0 & 4 & 0 & 2 & 0 \\
C & 0 & 2 & 2 & 0 & 2 \\
D & 0 & 0 & 0 & 4 & 2 \\
E & 0 & 0 & 0 & 2 & 2 \\
\hline
\end{tabular}

The data into the plot and rear calculated maintainability index of $\mathrm{H}$

$$
\begin{aligned}
\operatorname{per}(H) & =v_{1} v_{3} v_{2}\left(v_{4} v_{5}+4\right) \\
& =2 \bullet 2 \bullet 4(4 \bullet 2+4) \\
& =192
\end{aligned}
$$

The data into the plot and rear calculated $\mathrm{K}$ maintainability index

$$
\begin{aligned}
\operatorname{per}(K) & =v_{1} v_{3} v_{2}\left(v_{4} v_{5}+4\right) \\
& =2 \bullet 2 \bullet 2(4 \bullet 2+4) \\
& =96
\end{aligned}
$$


Table $6 \mathrm{~K}$ maintainability comprehensive weight matrix

\begin{tabular}{cccccc}
\hline $\begin{array}{c}\text { Indicators } \\
\text { / Score }\end{array}$ & A & B & C & D & E \\
\hline A & 2 & 4 & 2 & 4 & 2 \\
B & 0 & 2 & 0 & 2 & 0 \\
C & 0 & 2 & 2 & 0 & 2 \\
D & 0 & 0 & 0 & 2 & 2 \\
E & 0 & 0 & 0 & 2 & 2
\end{tabular}

Table $7 \mathrm{Z}$ maintainability comprehensive weight matrix

\begin{tabular}{cccccc}
$\begin{array}{c}\text { Indicators / } \\
\text { Score }\end{array}$ & A & B & C & D & E \\
\hline A & 2 & 4 & 2 & 4 & 2 \\
B & 0 & 4 & 0 & 2 & 0 \\
C & 0 & 2 & 2 & 0 & 2 \\
D & 0 & 0 & 0 & 0 & 2 \\
E & 0 & 0 & 0 & 2 & 4 \\
\hline
\end{tabular}

The data into the plot and rear calculated $Z$ maintainability index

$$
\begin{aligned}
\operatorname{per}(Z) & =v_{1} v_{3} v_{2}\left(v_{4} v_{5}+4\right) \\
& =2 \bullet 4 \bullet 2(0 \bullet 4+4) \\
& =64
\end{aligned}
$$

\subsection{Evaluation calculate the degree of influence of the components}

For the maintenance of the level of influence of the system, each member has a different role in weight. Since the degree of influence the size of the components is determined by the relative failure rate, so the right to re-determine the importance of component parts to the level of their failure rate as the basis. By fielded weapon systems and equipment maintenance records analyzed with units, combined with similar data obtained equipment failure frequency components of each weapon system. Because the sample data acquisition is large enough, so that the frequency of failure of each component is similar to its own failure rate. To facilitate data processing, the paper will be seen as failure rate failure frequency. For fire system $(\mathrm{H})$, the fire control system $(\mathrm{K})$, an integrated information system $(Z)$ is the failure rate of the statistical analysis to obtain a normalized failure rate of components, namely the weight value $\left(W_{H}, W_{K}, W_{Z}\right)$ :

$$
\begin{gathered}
W_{H}=\frac{\lambda_{h}}{\lambda_{h}+\lambda_{k}+\lambda_{z}} \\
W_{K}=\frac{\lambda_{k}}{\lambda_{h}+\lambda_{k}+\lambda_{z}} \\
W_{Z}=\frac{\lambda_{z}}{\lambda_{h}+\lambda_{k}+\lambda_{z}}
\end{gathered}
$$

The impact $\mathrm{H}, \mathrm{K}, \mathrm{Z}$ three components of maintainability index weapons systems are different. Each of the components with respect to the right to re-use of weapons systems components engineering experience data to the level of each of the components used to determine the failure rate [10] as the basis. The maintenance experience data collection, to get the right parts the importance of the various components as shown in Table 8 after heavy failure rate normalization will be shown

Right importance of each of the components in 
Table 8 heavy weapons systems

\begin{tabular}{cccc}
\hline Components & $\mathrm{H}$ & $\mathrm{K}$ & $\mathrm{Z}$ \\
\hline $\begin{array}{c}\text { importance } \\
\text { weights }\end{array}$ & 0.352 & 0.542 & 0.106 \\
\hline
\end{tabular}

Table 8 system components combined weights of each composition, the maintenance of substituting each component maintainability index calculated composite index for weapons systems

$$
\begin{aligned}
\operatorname{per}(W Q) & =W_{H} \operatorname{per}(H)+W_{K} \operatorname{per}(K)+W_{Z} \operatorname{per}(Z) \\
& =0.352 \times 192+0.542 \times 96+0.106 \times 64 \\
& =126.4
\end{aligned}
$$

Taking a member $(m)$, repair of the evaluation $i$, for example, set it in the context of the matrix score for the maintenance level $N$, out of the ideal state maintenance level score $N_{\max }$, the system's maintainability index for

$$
\operatorname{per}(W Q)=\sum_{\text {com }} W_{\text {com }} \operatorname{per}(\mathrm{com}) \quad(3)
$$

Defining equation (3) $c o m$ for the collection $\{H, K, Z\}$. The $i$ index score of 10 components $m$, the maintenance of the system should be as comprehensive index

$$
\operatorname{per}^{\prime}(W Q)=W_{m} \operatorname{per}(m)_{N_{\max }}+\sum_{\text {com }}^{m \neq c o m} W_{m} \operatorname{per}(\mathrm{com})
$$

Substituting into equation (1), the indicator member of the system maintenance level of the degree of influence $\Delta$ of

$$
\Delta=\frac{W_{m} \operatorname{per}(m)_{N_{\max }}+\sum_{\text {com }}^{\text {mecom }} W_{m} \operatorname{per}(\text { com })-\sum_{\text {com }} W_{\text {com }} \operatorname{per}(\text { com })}{\sum_{\text {com }} W_{\text {com }} \operatorname{per}(\mathrm{com})}
$$

Changes in the system to enhance maintainability index components by a specific grade level of maintainability evaluation brought that influence the degree of evaluation, as shown in Table 9

Table 9 Evaluation affect each of the components of the

\begin{tabular}{cccccc} 
& $\mathrm{A}$ & $\mathrm{B}$ & $\mathrm{C}$ & $\mathrm{D}$ & $\mathrm{E}$ \\
\hline $\mathrm{H}$ & $\Delta_{H}^{A}$ & $\Delta_{H}^{B}$ & $\Delta_{H}^{C}$ & $\Delta_{H}^{D}$ & $\Delta_{H}^{E}$ \\
$\mathrm{~K}$ & $\Delta_{K}^{A}$ & $\Delta_{K}^{B}$ & $\Delta_{K}^{C}$ & $\Delta_{K}^{D}$ & $\Delta_{K}^{E}$ \\
$\mathrm{Z}$ & $\Delta_{Z}^{A}$ & $\Delta_{Z}^{B}$ & $\Delta_{Z}^{C}$ & $\Delta_{Z}^{D}$ & $\Delta_{Z}^{E}$ \\
\hline
\end{tabular}

\section{4. calculation results}

When calculated separately for each component maintainability index score of 10 (ie, without considering the impact on the maintenance of the level of the index), the system maintainability index to fire system $(\mathrm{H})$ Maintainability Evaluation accessibility (A) score of 4 when an example, $\mathrm{H}$ maintainability index components are:

$$
\begin{aligned}
\operatorname{per}^{A}(H) & =v_{1} v_{3} v_{2}\left(v_{4} v_{5}+4\right) \\
& =4 \bullet 2 \bullet 4(2 \bullet 4+4) \\
& =256
\end{aligned}
$$

The system maintainability index is: 


$$
\begin{aligned}
& \operatorname{per}_{H}^{A}(W Q) \\
& =W_{H^{*}} \operatorname{per}^{A}(H)+W_{K^{*}} \operatorname{per}(K)+W_{Z^{*}} \operatorname{per}(Z) \\
& =0.352 \times 256+0.542 \times 96+0.106 \times 64 \\
& =148.9
\end{aligned}
$$

Into (1)

$$
\Delta_{H}^{A}=\frac{\operatorname{per}_{H}^{A}(W Q)-\operatorname{per}(W Q)}{\operatorname{per}(W Q)}
$$

A calculated index $\mathrm{H}$ component of the system maintenance level of influence degree $\Delta_{H}^{A}=0.178$ And so each of the components of the evaluation of the impact of weapon system maintenance level when different indicators were calculated out of the various components, as shown in Table 10

Table 10 Maintenance of various components of the degree of influence evaluation

\begin{tabular}{cccccc}
\hline & $\mathrm{A}$ & $\mathrm{B}$ & $\mathrm{C}$ & $\mathrm{D}$ & $\mathrm{E}$ \\
\hline $\mathrm{H}$ & 0.178 & 0 & 0.352 & 0 & 0.235 \\
$\mathrm{~K}$ & 0.542 & 0.542 & 0.542 & 0 & 0.361 \\
$\mathrm{Z}$ & 0.106 & 0.106 & 0 & 0.424 & 0
\end{tabular}

The resulting data drawn from Table 10 Evaluation of the system maintainability levels shown in Figure 2

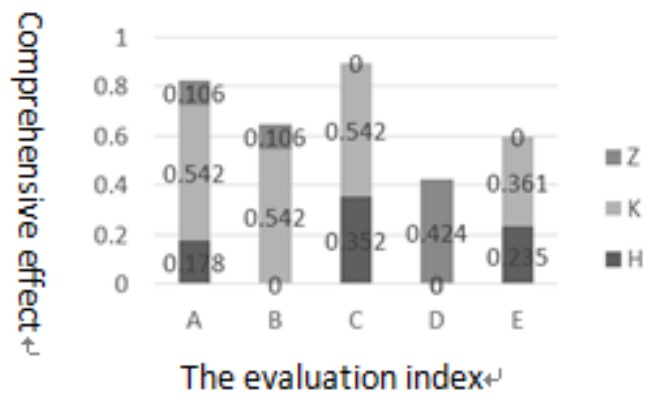

Figure 2 maintainability evaluation components change the influence diagram

As can be seen from Figure 2: Effect of changes in C indicators weapon system maintenance level of the maximum, ie its role in promoting appropriate to improve the design of the system maintenance level of the best, so when you want to focus on improving the design of this consideration factors. D Indexes degree of influence on the maintenance of a minimum weapon system, and the degree of influence on the $\mathrm{H}, \mathrm{K}$ component is $0, \mathrm{D}$ indicators in the $\mathrm{H}, \mathrm{K}$ Components has almost no room for improvement, and improve the design of $\mathrm{H}$, the $\mathrm{K}$ Components do not need to be modified to consider upgrading. Overall, the system is to enhance the level of service a larger space, indicating their relative maintenance level is not high.

\section{Conclusion}

On the basis of considering the various components of weapon systems weights and evaluation of the correlation degree, the degree of influence by calculating the index comprehensive evaluation of the weapon system maintainability. This evaluation method is not limited to the maintenance of the composite index of solving the component level, but get the system-level maintenance of the composite index by decomposing combination of the way, and the degree of influence of each index were calculated, reflecting the idea of systems engineering . Through a comparative analysis on the degree of influence can accurately determine the components as well as the evaluation of the weapon system maintenance level extent size, the quantitative evaluation of the level of maintainability. Using this method you can also get the level of maintenance to improve the design of each index of room for improvement, the relative gap between the system's own desired level of 
information, and promoting the growth of weapon system maintainability.

\section{References}

[1] CAI Jing, Zuo Hongfu, Liu et Complex Systems Group Maintenance strategy optimization model [J] Journal of Applied Sciences, 2006, vol. 24 (Section 5): 533-537.

[2] Yan Pengfei, Sun Quan weighted fusion method based on confidence in the maintenance of the application evaluation [J] Computer Simulation, 2010, vol. 27 (Section 6): 31-35.

[3] Chen Lu, Jiang Dandong, Cai Jianguo product maintenance and implementation of the evaluation model algorithm [J]. China Mechanical Engineering, 2003, vol. 14 (Article 21): 2712-2716

[4] Zeng Changsha reachability analysis based on the evaluation of maintainability design technology research [D]: National University of Defense Technology, 2007.

[5] von Bounded Model Checking studies on the weapon system maintainability design evaluation methods [J] Ordnance Sinica, 2003, 24 (2): 222-225

[6] Ding Yong, Yangzi Jia, Zhou Wei Jie Research virtual civil aircraft maintenance products evaluation method [J] aircraft design, 2012,32 (1): 72-77

[7] JU Gui-zhi, ZHAO Ya-qun Correlation Degree and Correlation Coefficient of Multi-Output Functions [J] Wuhan University Journal of Natural Sciences, 2005, 10 (2): 195-198

[8] Maintainability Verification Demonstration / Evaluation MIL-STD-471A (US)

[9] Liu Qi, Wu Xiaoyue integrated analysis model for complex system reliability growth test evaluation [J] Systems Engineering Theory and Practice, 2010, 30 (8): 1477-1482

[10] Lu, the SUN study towards virtual product maintainability evaluation model based on Fuzzy Multiple Attribute Decision Making Theory [J]. China Mechanical Engineering, 2007,20 (24): 2978-2983 